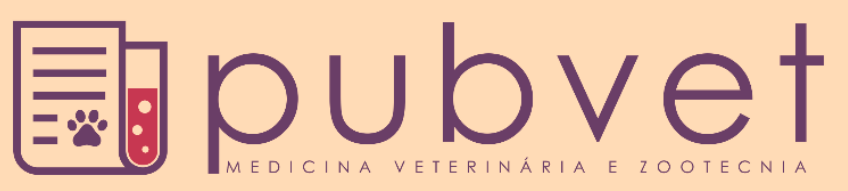

https://doi.org/10.31533/pubvet.v16n02a1049.1-6

\title{
Perfil populacional de animais para adoção em organização não governamental (ONG) de Joinville, Santa Catarina
}

\author{
Julia de Freitas ${ }^{1 *}$, Carlize Lopes $^{29}$, Carlos Eduardo Nogueira Martins ${ }^{2 \oplus}$ \\ ${ }^{1}$ Discente de Medicina Veterinária do Instituto Federal Catarinense - Campus Araquari - Santa Catarina, Brasil. \\ ${ }^{2}$ Professor(a) do Instituto Federal Catarinense - Campus Araquari - Santa Catarina, Brasil. \\ *Autor para correspondência, E-mail: judfreitass@gmail.com
}

\begin{abstract}
Resumo. Dados apontam um elevado número de animais errantes no Brasil. Os abrigos trabalham na tentativa de conter o aumento de animais abandonados, fornecendo um local adequado para permanência temporária e promovendo ações de conscientização da população local. O propósito deste estudo foi avaliar o perfil populacional de animais na instituição na tentativa de compreender as características de abandono e influência do abrigo na comunidade. Para elaboração deste trabalho foram utilizados dados pertencentes a uma organização não governamental da cidade de Joinville, Santa Catarina. Foram solicitadas informações referentes a características dos animais abrigados, como espécie, sexo, idade, status vacinal e reprodutivo. Os dados foram planilhados e submetidos à análise estatística. Os resultados mostraram um predomínio da população de cães em relação aos gatos. Também pode ser observado um reduzido número de animais com idade inferior a 12 meses em ambas as espécies. Quanto ao sexo, houve diferença apenas em caninos, onde o número de machos foi superior ao de fêmeas. De acordo com o protocolo da instituição, todos os animais foram submetidos a vacinação assim que admitidos e encaminhados a castração no momento que estiverem aptos para o procedimento cirúrgico. Campanhas de adoção e o incentivo à guarda responsável são mecanismos utilizados pela ONG para conscientizar a população e encontrar um lar para os animais abrigados aumentando a sua capacidade de acolhimento. A diferença na proporção de animais entre as espécies, sexo e idade tem relação direta com as características buscadas pelos adotantes. Os dados evidenciam a importância dos abrigos na diminuição dos animais errantes e promoção do bem-estar animal, sendo irrefutável a necessidade do incentivo e manutenção dessas instituições. Também é fundamental a elaboração de políticas públicas que assegurem o acesso a serviço veterinário em todas as localidades para a população impossibilitada de custear o tratamento de seus animais de estimação.
\end{abstract}

Palavras-chave: Abandono, guarda responsável, maus-tratos, saúde pública

\section{Population profile of animals for adoption in a non-governmental organization (NGO) in Joinville, Santa Catarina}

Abstract. Data show a high number of stray animals in Brazil. The shelters work in an
attempt to contain the increase in abandoned animals, providing a suitable place for
temporary stay and promoting awareness actions for the local population. The purpose of
this study was to evaluate the population profile of animals in the institution in an attempt
to understand the characteristics of abandonment and influence of the shelter on the
community. For the elaboration of this work, data belonging to a non-governmental
organization in the city of Joinville, Santa Catarina, were used. Information was requested
regarding characteristics of the sheltered animals, such as species, sex, age, vaccination and
reproductive status. Data were spreadsheets and submitted to statistical analysis. The 
results showed a predominance of the dog population over cats. A reduced number of animals under 12 months of age can also be observed in both species. As for sex, there was a difference only in canines, where the number of males was higher than that of females. According to the institution's protocol, all animals undergo vaccination as soon as they are admitted and sent to castration at the time they are ready for the surgical procedure. Adoption campaigns and the encouragement of responsible custody are mechanisms used by the NGO to raise awareness among the population and find a home for the sheltered animals, increasing their capacity for shelter. The difference in the proportion of animals between species, sex and age is directly related to the characteristics sought by adopters. The data show the importance of shelters in reducing stray animals and promoting animal welfare, and the need for encouragement and maintenance of these institutions is irrefutable. It is also essential to develop public policies that ensure access to veterinary services in all locations for the population unable to pay for the treatment of their pets.

Key words: Abandonment, responsible guard, mistreatment, public health

\section{Perfil poblacional de animales en adopción en una organización no gubernamental (ONG) en Joinville, Santa Catarina}

Resumen. Los datos muestran una gran cantidad de animales callejeros en Brasil. Los albergues trabajan en un intento por contener el aumento de animales abandonados, proporcionando un lugar adecuado para la estancia temporal y promoviendo acciones de sensibilización para la población local. El propósito de este estudio fue evaluar el perfil poblacional de animales en la institución en un intento por comprender las características del abandono y la influencia del refugio en la comunidad. Para la elaboración de este trabajo se utilizaron datos pertenecientes a una organización no gubernamental de la ciudad de Joinville, Santa Catarina. Se solicitó información sobre las características de los animales protegidos, como especie, sexo, edad, vacunación y estado reproductivo. Los datos fueron hojas de cálculo y se sometieron a análisis estadísticos. Los resultados mostraron un predominio de la población canina sobre la felina. También se puede observar un número reducido de animales menores de 12 meses en ambas especies. En cuanto al sexo, solo hubo diferencia en caninos, donde el número de machos fue mayor que el de hembras. De acuerdo con el protocolo de la institución, todos los animales fueron vacunados tan pronto fueron admitidos y enviados a castración cuando estavan listos para el procedimiento quirúrgico. Las campañas de adopción y el fomento de la custodia responsable son mecanismos que utiliza la ONG para concienciar a la población y encontrar un hogar para los animales resguardados, aumentando su capacidad de refugio. La diferencia en la proporción de animales entre especies, sexo y edad está directamente relacionada con las características buscadas por los adoptantes. Los datos muestran la importancia de los refugios para reducir los animales callejeros y fomento del bienestar animal, y la necesidad de incentivar y mantener estas instituciones es irrefutable. También es fundamental desarrollar políticas públicas que aseguren el acceso a los servicios veterinarios en todas las localidades a la población que no pueda pagar el tratamiento de sus mascotas.

Palabras clave: Abandono, custodia responsable, malos tratos, salud pública

\section{Introdução}

O processo de domesticação dos animais instituiu uma relação instrumental e afetiva que perdura até hoje (Cabral \& Savalli, 2020). Segundo Giumelli \& Santos (2016), apesar da aproximação, a relação permanece sendo caracterizada pelo autoritarismo, onde o homem define quanto a liberdade dos animais. Em 2020, a Organização Mundial da Saúde (OMS), estimou cerca de 30 milhões de animais em situação de rua no Brasil, sendo sua maioria cães (Martinhago \& Magalhães, 2018).

Neste contexto, destaca-se o conceito de saúde única, a qual é definida como o equilíbrio na relação entre humanos, animais e o ambiente (Carvalho \& Pessanha, 2013; Sousa \& Silva, 2012). O contato próximo entre as espécies possibilitou o aparecimento de doenças conhecidas como zoonoses, comum 
entre humanos e animais (Beserra et al., 2020; Schantz, 1991). Além das zoonoses, a superpopulação de animais nas ruas, aumenta a ocorrência de maus-tratos, acidentes e mordeduras (Marlet \& Maiorka, 2010). Também ocasiona falta de água, alimento, abrigo e os deixam suscetíveis a diversas doenças (Paula et al., 2018).

A adoção é fundamental para a diminuição de animais em situação de vulnerabilidade. Associada à ela, é essencial o incentivo à guarda responsável, onde as famílias são instruídas sobre a importância de proporcionar níveis adequados de bem-estar (Jorge et al., 2018; Oliveira, 2016; Santana \& Oliveira, 2006). Por outro lado, muitos animais de rua são encaminhados para diferentes instituições. Os abrigos podem ser de caráter governamental ou não, públicos, privados ou mistos e possuir um papel importante no acolhimento desses animais (Ouriques, 2018). Preservam o bem-estar animal, em sua maioria, ao proporcionar um ambiente adequado, fornecer assistência veterinária, realizar castrações e vacinações, promover campanhas de conscientização, ressocializar e os reintroduzir na sociedade (Arruda et al., $\underline{2019}$; $\underline{\text { Creevy et al., 2019). }}$

O presente estudo teve como objetivo avaliar o perfil populacional dos animais para adoção em abrigo localizado na cidade de Joinville, Santa Catarina, identificando as características dos animais abandonados e o papel da ONG na comunidade.

\section{Material e métodos}

Foram obtidos dados referentes ao perfil populacional dos animais disponíveis para adoção em uma organização não governamental (ONG) localizada na cidade de Joinville (26 $16^{\circ} 18^{\prime \prime} \mathrm{S} 48^{\circ} 50^{\prime} 46^{\prime \prime} \mathrm{O}$ ), Santa Catarina. A associação Abrigo Animal é uma organização não governamental sem fins lucrativos fundada em julho de 2001. Em 20 de junho de 2018 foi firmado convênio com a Prefeitura Municipal de Joinville (Secretaria de Agricultura e Meio Ambiente) onde o Centro de Bem-Estar Animal (CBEA) transfere os animais aptos para adoção para o local.

A coleta de dados foi realizada indiretamente por um checklist elaborado e enviado a representante da entidade Abrigo Animal para que fossem disponibilizadas as informações contidas no banco de dados da instituição a respeito do mês de junho de 2021. Para tal, foi solicitado o número de animais no local, número de animais por espécie, número de animais com idade inferior e superior a 12 meses, número de animais machos e fêmeas por espécie e o número de animais castrados e vacinados por espécie.

Os dados obtidos foram inseridos em uma planilha no Microsoft Excel para categorização e submetidos ao teste do Qui-quadrado, sendo considerado o nível de significância de $\mathrm{P}<0,05$ para todas as análises. Para tal, foi utilizado o programa estatístico (JAMOVI, 2021).

\section{Resultados e discussão}

Segundo o Instituto Brasileiro de Geografia e Estatística, a população de Joinville estimada em 2020 foi de 515 mil habitantes, sendo considerada a cidade mais populosa do estado, possuindo uma unidade territorial de $1.127,946 \mathrm{~km}^{2}$ (IBGE, 2020).

No mês de junho de 2021 estavam abrigados na organização 326 animais, havendo diferença $(\mathrm{P}<$ $0,05)$ entre a proporção de cães e gatos (Figura 1).

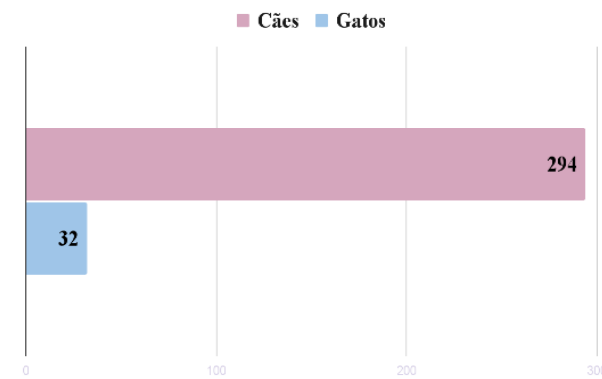

Figura 1. Número de cães e gatos abrigados em uma Organização Não Governamental de Joinville, Santa Catarina, no mês de junho de 2021. 
A grande maioria dos animais no abrigo, tanto para cães quanto gatos, tinha idade superior a 12 meses (Tabela 1). Tal fato corrobora com a pesquisa realizada por Moutinho et al. (2019), onde a maioria dos animais adotados possuíam idade inferior a 12 meses de vida, indicando maior facilidade das instituições de encontrar tutores para esses animais em comparação com aqueles com idade superior a 12 meses.

As vagas nos abrigos dependem, principalmente, do número de adoções. O tempo de permanência prolongado nas instituições compromete a capacidade de acolhimento, podendo levar a superpopulação e escassez de recursos. Fator que dificulta a adoção de animais adultos, e principalmente, idosos, é a inexistência em maioria das cidades de um serviço público veterinário. Sabe-se, que animais idosos raramente são acometidos por apenas uma doença e necessitam de acompanhamento veterinário mais frequente, quando comparado com filhotes e adultos jovens, para que problemas médicos e comportamentais sejam detectados no seu estágio mais precoce (Goldston \& Hoskins, 1999; $\underline{\text { Hoskins, 2008). }}$.

Tabela 1. Idade dos cães e gatos abrigados em uma Organização Não Governamental de Joinville, Santa Catarina, no mês de Junho de 2021

\begin{tabular}{lccc}
\hline Idade, meses & Cães & Gatos & Total \\
\hline$<12$ & 4 & 2 & 6 \\
$>12$ & 290 & 30 & 320 \\
Total & 294 & 32 & 326 \\
\hline
\end{tabular}

Quanto ao sexo, houve predominância de cães machos em relação a fêmeas (Tabela 2). Machos e fêmeas, assim como filhotes e adultos, são populações diferentes com comportamentos distintos e a escolha entre eles está diretamente relacionada com a preferência do adotante (Pastori \& Matos, 2015).

Tabela 2. Número de machos e fêmeas, por espécie, abrigados em uma Organização Não Governamental de Joinville, Santa Catarina, em junho de 2021

\begin{tabular}{lccc}
\hline Sexo & Cães & Gatos & Total \\
\hline Machos & 206 & 16 & 222 \\
Fêmeas & 88 & 16 & 104 \\
Total & 294 & 32 & 326 \\
\hline
\end{tabular}

Dos 326 animais no abrigo, 318 haviam sido castrados. Apenas duas fêmeas, uma lactante e uma gestante, e os 6 animais com idade inferior a 12 meses aguardavam a castração. Todos os animais, assim que acolhidos, foram vacinados e, se possível, encaminhados para a castração. Tutores de filhotes que não se encontram aptos para o procedimento cirúrgico são instruídos a contatarem a instituição assim que $o$ animal atingir a idade adequada.

Não há consenso sobre a idade adequada para realização da castração. Estudos apontam prós e contras tanto do procedimento precoce, quanto tardio. Na maioria dos casos é recomendado, para fêmeas, a castração entre o primeiro e segundo cio, em cadelas, e até os 12 meses de vida em gatas, de modo a auxiliar o controle populacional evitando gestações indesejadas, diminuindo a probabilidade de desenvolvimento de neoplasias mamária, evitando casos de piometra e outras patologias. Para machos, a castração precoce é indicada para o controle de animais errantes e prevenção de hiperplasia prostática, neoplasias testiculares, torção do cordão espermático, entre outras. Em ambos os sexos a castração está relacionada com o aumento da expectativa de vida e deve ser amplamente incentivada para fins de diminuição do número de animais em situação de vulnerabilidade (Alves \& Hebling, 2020).

Tanto os cães quanto os gatos receberam a vacina antirrábica. A raiva é uma enfermidade viral e zoonótica que afeta o sistema nervoso central, de curso fatal em quase sua totalidade de casos. Estimase que cerca de $95 \%$ dos casos em humanos são decorrentes de mordidas de cães infectados (Quinn et al., 2018). Para cães foi realizada, também, a vacinação contra a cinomose, parvovirose, adenovirose, coronavirose, parainfluenza e leptospirose. E em gatos contra panleucopenia, calicivirose, rinotraqueíte e clamidiose.

A superpopulação de animais de rua é um grave problema para a saúde pública. Sabe-se, que cadelas e gatas podem ter vários filhotes em uma única gestação. Tal fato contribui significativamente para o aumento do número de animais errantes. Estes, são passíveis de contraírem e transmitirem doenças, sofrerem com ações de maus-tratos, acidentes, falta de água, comida e abrigo (Scherer et al., 2021). 
Dessa forma, a adoção de medidas como a castração e vacinação por instituições que acolhem esses animais é um importante mecanismo de promoção da saúde única. Ademais, pelas campanhas, os abrigos desempenham um papel social de incentivo à guarda responsável de animais domésticos, caracterizada pela ciência do tutor de sua função como cuidador e fornecendo níveis adequados de bemestar.

\section{Considerações finais}

O perfil populacional dos animais abrigados na instituição compreende, majoritariamente, animais com mais de 12 meses de vida, sendo em sua maioria caninos, machos, seguidos de fêmeas da mesma espécie. Quanto aos felinos, não houve diferença na proporção de machos e fêmeas. Animais com idade inferior a 12 meses de vida, de ambas as espécies, são encontrados em menor quantidade.

Sobretudo, entende-se como necessário o fomento e a preservação de instituições como abrigos, que acolhem animais em situação de abandono proporcionando um ambiente adequado para permanência até que estes sejam adotados, ou em diversos casos, até o final de suas vidas. Simultaneamente, é essencial o incentivo a adoção e a guarda responsável, para que esses animais errantes tenham a possibilidade de encontrar um lar que supra suas necessidades. Também, faz-se urgente políticas públicas que viabilizem o acesso a serviço veterinário em todas as localidades, com intuito de diminuir o número de abandonos ou eutanásias em decorrência da impossibilidade dos tutores arcarem com os custos dos tratamentos.

\section{Referências bibliográficas}

Alves, B. F. A., \& Hebling, L. M. G. F. (2020). Vantagens e desvantagens da castração cirúrgica de cães domésticos. Uma revisão integrativa de literatura. Brazilian Journal of Development, 6(9), $73157-$ 73168. https://doi.org/10.34117/bjdv6n9-683.

Arruda, E. C., Noronha, J., Molento, C. F. M., Garcia, R. C. M., \& Oliveira, S. T. (2019). Características relevantes das instalações e da gestão de abrigos públicos de animais no estado do Paraná, Brasil, para o bem-estar animal. Arquivo Brasileiro de Medicina Veterinária e Zootecnia, 71, 232-242. https://doi.org/10.1590/1678-4162-10224.

Beserra, E. E., Falcão, B. M. R., Nascimento, D. P., Ferreira, A. G. M., Rocha, R. C., \& Fernandes, A. R. F. (2020). COVID-19 e a atuação do médico veterinário no contexto da saúde única. Research, Society and Development, 9(10), e9069109411-e9069109411. https://doi.org/10.33448/rsdv9i10.9411.

Cabral, F. G. S., \& Savalli, C. (2020). La relation homme-chien. Psicologia USP, 31. https://doi.org/10.1590/0103-6564e190109.

Carvalho, R. L. S., \& Pessanha, L. D. R. (2013). Relação entre famílias, animais de estimação, afetividade e consumo: estudo realizado em bairros do Rio de Janeiro. Revista Sociais e Humanas, 26(3), 622-637. https://doi.org/10.5902/231717586562.

Creevy, K. E., Grady, J., Little, S. E., Moore, G. E., Strickler, B. G., Thompson, S., \& Webb, J. A. (2019). AAHA canine life stage guidelines. Journal of the American Animal Hospital Association, 55(6), 267-290. https://doi.org/10.5326/JAAHA-MS-6999.

Giumelli, R. D., \& Santos, M. C. P. (2016). Convivência com animais de estimação: um estudo fenomenológico. Revista Da Abordagem Gestáltica: Phenomenological Studies, 22(1), 49-58.

Goldston, R. T., \& Hoskins, J. D. (1999). Geriatria e gerontologia do cão e do gato. Rocca.

Hoskins, J. D. (2008). Geriatria e Gerontologia do cão e do gato. Editora Roca.

JAMOVI. (2021). The Jamoviprojet, VERSION 1.6.

Jorge, S., Barbosa, M. J., Wosiacki, S., \& Ferrante, M. (2018). Guarda responsável de animais: conceitos, ações e políticas públicas. Enciclopedia Biosfera, 15(28). https://doi.org/10.18677/EnciBio_2018B51.

IBGE (2020). Instituto Brasileiro de Geografia e Estatística (IBGE). Joinville, Santa Catarina, Brasil. IBGE. 2020. Disponível em: https://cidades.ibge.gov.br/brasil/sc/joinville/panorama.

Marlet, E. F., \& Maiorka, P. C. (2010). Análise retrospectiva de casos de maus tratos contra cães e gatos 
na cidade de São Paulo. Brazilian Journal of Veterinary Research and Animal Science, 47(5), 385 394. https://doi.org/10.11606/issn.1678-4456.bjvras.2010.26820.

Martinhago, S. S., \& Magalhães, T. A. P. (2018). A ineficácia das políticas públicas para o controle de animais de rua em Cascavel/PR. Diálogos e Interfaces do Direito, 1(1), 117-130.

Moutinho, F. F. B., Serra, C. M. B., \& Valente, L. C. M. (2019). Situação pós-adoção dos animais adotados junto a uma Ong de proteção dnimal no estado do Rio de Janeiro. Ciência Animal Brasileira, 20, 1-14. https://doi.org/10.1590/1809-6891v20e-43777.

Oliveira, A. B. (2016). Índice estatístico de animais domésticos resgatados da rua vs adoção. Revista Dimensão Acadêmica, 1(2), 1-14.

Ouriques, J. R. (2018). Bem estar animal: Um abrigo para cães e gatos vítimas de maus-tratos e abandono em Florianópolis. In Arquitetura-Florianópolis. Universidade do Sul de Santa Catarina.

Pastori, É. O., \& Matos, L. G. (2015). Da paixão à "ajuda animalitária": o paradoxo do "amor incondicional" no cuidado e no abandono de animais de estimação. Caderno Eletrônico de Ciências Sociais, 3(1), 112-132. https://doi.org/10.24305/cadecs.v3i1.12277.

Paula, J. M., Santos, C. G., Canalli, V., Fritzen, D. M. M., Busato, M. A., \& Lutinski, J. A. (2018). Perfil populacional de cães e gatos e bem-estar animal em Chapecó, SC. Revista Brasileira de Higiene e Sanidade Animal, 12(4), 437-449. https://doi.org/10.5935/1981-2965.20180040.

Quinn, P. J., Markey, B. K., Leonard, F. C., Fitzpatrick, E. S., \& Fanning, S. (2018). Microbiologia veterinária essencial. Artmed Editora.

Santana, L. R., \& Oliveira, T. P. (2006). Guarda responsável e dignidade dos animais. Revista Brasileira de Direito Animal, 1, 207-230.

Schantz, P. M. (1991). Parasitic zoonoses in perspective. International Journal for Parasitology, 21(2), $161-170$.

Scherer, A., Laureano, D. B., Andrade, E. V. R., Freitas, J. F., Braghiroli, N., Silva, S. G., \& Mendonça, R. C. (2021). A importância da adoção de animais no Brasil. PUBVET, 15(7), 1-5. https://doi.org/10.31533/pubvet.v15n07a872.1-5.

Sousa, M. R. Q., \& Silva, F. B. S. (2012). Interação homem-animal e sua relação com a guarda responsável de cães em um bairro da cidade do Recife-PE. PUBVET, 6(5), 1-13. https://doi.org/10.22256/pubvet.v16n5.1294.

Histórico do artigo

Recebido: 16 de setembro de 2021

Aprovado: 27 de outubro de 2021

Disponível online: 28 de fevereiro de 2022
Licenciamento: Este artigo é publicado na modalidade Acesso Aberto sob a licença Creative Commons Atribuição 4.0 (CC-BY 4.0), a qual permite uso irrestrito, distribuição, reprodução em qualquer meio, desde que o autor e a fonte sejam devidamente creditados. 